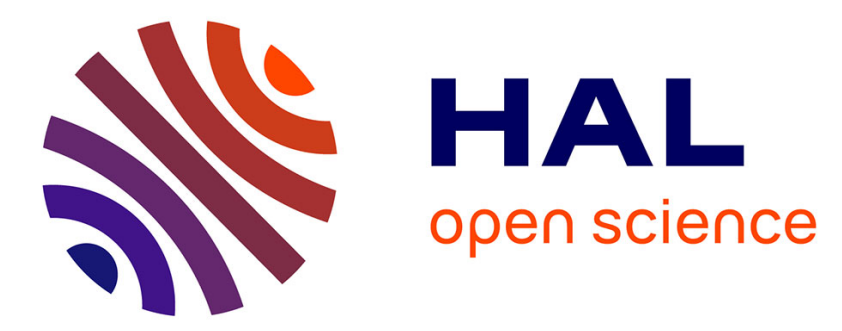

\title{
Image-based estimation of myocardial acceleration using TDFFD: a phantom study
}

Ali Pashaei, Gemma Piella, Nicolas Duchateau, Luigi Gabrielli, Oscar Camara

\section{To cite this version:}

Ali Pashaei, Gemma Piella, Nicolas Duchateau, Luigi Gabrielli, Oscar Camara. Image-based estimation of myocardial acceleration using TDFFD: a phantom study. Statistical Atlases and Computational Modeling of the Heart (STACOM 2013), 2013, Nagoya, Japan. pp.262-270, 10.1007/978-3-64254268-8_31. hal-02320194

\section{HAL Id: hal-02320194 \\ https://hal.science/hal-02320194}

Submitted on 18 Oct 2019

HAL is a multi-disciplinary open access archive for the deposit and dissemination of scientific research documents, whether they are published or not. The documents may come from teaching and research institutions in France or abroad, or from public or private research centers.
L'archive ouverte pluridisciplinaire HAL, est destinée au dépôt et à la diffusion de documents scientifiques de niveau recherche, publiés ou non, émanant des établissements d'enseignement et de recherche français ou étrangers, des laboratoires publics ou privés. 


\title{
Image-based estimation of myocardial acceleration using TDFFD: a phantom study
}

\author{
Ali Pashaei ${ }^{1}$, Gemma Piella ${ }^{1}$, Nicolas Duchateau ${ }^{2}$, Luigi Gabrielli ${ }^{2,3}$, and \\ Oscar Camara ${ }^{1}$ \\ ${ }^{1}$ Universitat Pompeu Fabra, Barcelona, Spain \\ 2 Hospital Clínic, IDIBAPS, Universitat de Barcelona, Spain \\ ${ }^{3}$ Pontificia Universidad Católica de Chile, Santiago, Chile
}

\begin{abstract}
In this paper, we propose to estimate myocardial acceleration using a temporal diffeomorphic free-form deformation (TDFFD) algorithm. The use of TDFFD has the advantage of providing B-spline parameterized velocities, thus temporally smooth, which is an asset for the computation of acceleration. The method is tested on $3 \mathrm{D}+\mathrm{t}$ echocardiographic sequences from a realistic physical heart phantom, in which ground truth displacement is known in some regions. Peak endocardial acceleration (PEA) error was $20.4 \%$, the main hypothesis for error being the low temporal resolution of the sequences. The allure of the acceleration profile was reasonably preserved. Our method suggests a noninvasive technique to measure cardiac acceleration that may be used to improve the monitoring of cardiac mechanics and consecutive therapy planning.
\end{abstract}

\section{Introduction}

Several studies have shown that cardiac acceleration such as peak endocardial (PEA) and epicardial acceleration can be used as an index to assess function of the heart (such as max dp/dt) and detection of myocardial ischemia [1, 2]. For this reason, accelerometer devices have been proposed for monitoring myocardial ischemia and cardiac resynchronization therapy (CRT) optimization [2, 3, 4]. However, these measurements are highly invasive, and only available at a singlelocation. Also, it was indicated that accelerometer measurements are influenced by patient orientation and gravity $[5,6]$.

Image-based computation of cardiac acceleration has been recently proposed, which may resolve these issues [7]. This study computed the acceleration directly from the displacement fields estimated by an image-based registration algorithm [8].

In the present work, we address this issue by computing acceleration directly from the parametric differentiation of myocardial velocities, the estimation of which is inherent to the registration process. The use of temporal diffeomorphic free-form deformation (TDFFD) algorithm has the advantage of providing Bspline parameterized velocities, thus spatiotemporally smooth, which is an asset for the computation of acceleration directly from these velocities. We extend 


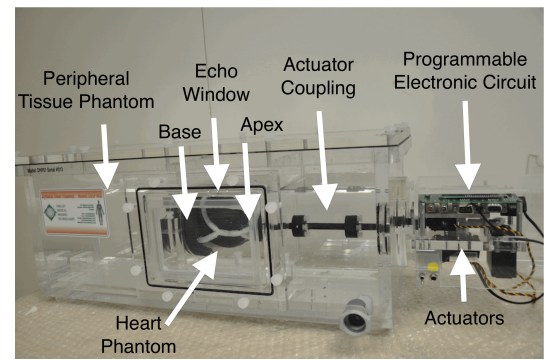

(a)

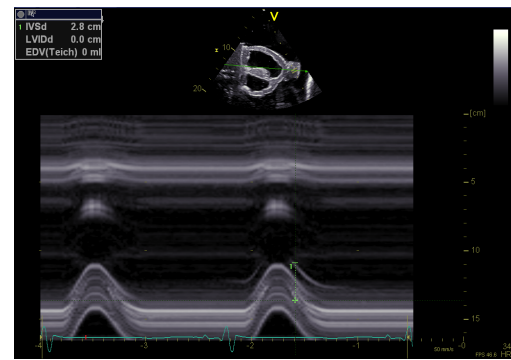

(b)

Fig. 1. (a) Dynamic heart phantom, and (b) M-mode data showing the sinus waveform displacement function of equation (1) generated by the phantom.

the evaluation of the accuracy of such computations using data from a realistic physical heart phantom, for which ground truth is known at specific locations.

\section{Materials and Methods}

\subsection{Imaging data}

A dynamic multimodality heart phantom (DHP-01, Shelley Medical Imaging Technologies, London, ON, CA) was employed to provide data for computation of the cardiac acceleration from images. This phantom mimics realistic anatomical geometry of the left and right ventricles of the human heart. The dynamic heart phantom setup is shown in Fig. 1(a).

This phantom has two controlable actuation systems for translational and rotational movement. Translation phantom actuator (located at the apex) was programmed to follow a sinus waveform, without any rotation. The function for the movement of actuator was:

$$
\mathbf{d}_{\text {apex }}=\frac{A}{2}(1-\cos (2 \pi t / T)),
$$

hence providing the acceleration as;

$$
\mathbf{a}_{\text {apex }}=\frac{A}{2}\left(\frac{2 \pi}{T}\right)^{2} \cos (2 \pi t / T) .
$$

The actuator was set to have stroke value $A=0.02 \mathrm{~m}$ within a period of $T=1.05 \mathrm{sec}$. The movement contained a short delay after each movement, hence providing a heart rate of $34 \mathrm{bpm}$.

Fig. 1(b) shows 2D M-mode visualization of the programmed phantom motion, for qualitative checking purposes.

$3 \mathrm{D}+\mathrm{t}$ echocardiographic sequences were acquired for the acceleration computations. Fig. 2 shows a representation of the acquired 3D data. Animated 


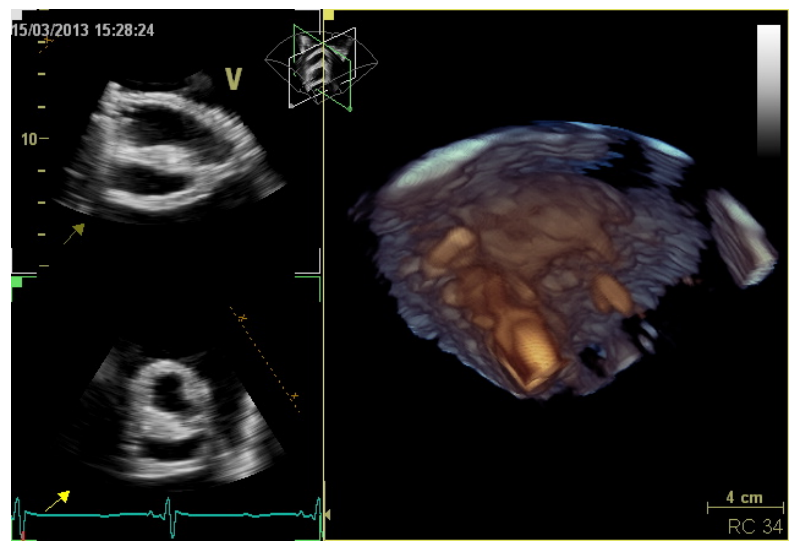

Fig. 2. Acquired 3D data at end-diastole.

version of this figure is available online here. The difference with real data acquisitions is that the phantom is surrounded by water inside and outside its walls, contrary to clinical images where tissue is visible outside the cardiac cavities, and therefore can make the image quality lower and the estimation of wall motion more difficult. Two temporal resolutions (11.9 and $21.7 \mathrm{fps}$ ) were used for $3 \mathrm{D}+\mathrm{t}$ echocardiographic acquisition. All images were acquired with gating to a simulated vector ECG.

\section{$2.2 \quad$ Image processing}

Segmentation of the left and right ventricles was first performed using 3D Active Shape Models (ASM) [9]. The mesh resulting from the segmentation was matched to the $3 \mathrm{D}+\mathrm{t}$ echocardiographic sequences and propagated along the cycle by means of a non-rigid image registration algorithm (TDFFD) [8]. We used a two-level multiresolution implementation of the TDFFD. The initial grid size was of one control point per frame in the temporal direction, 5 control points in the short-axis direction and in the long-axis direction. As similarity measure, we used the sum of squared differences between the intensities of each frame and a reference (end-diastole) one. We used the L-BFGS-B as optimizer [10]. The left ventricle was divided using the 17-segment model as proposed by the American Heart Association (AHA) [11].

\subsection{Computation of acceleration}

Acceleration is computed as the derivative of the estimated velocity function, which is defined in terms of B-Spline kernels. Here we modified this function to 
estimate acceleration directly from the TDFFD output. If the B-Spline coefficients of all contol points are presented in a vector of parameters $\mathbf{p}$, the velocity $\mathbf{v}(\mathbf{x}, t ; \mathbf{p})$ is computed as

$$
\mathbf{v}(\mathbf{x}, t ; \mathbf{p})=\sum_{i, j, k, l} \beta\left(\frac{x-x_{i}}{\Delta_{x}}\right) \beta\left(\frac{y-y_{j}}{\Delta_{y}}\right) \beta\left(\frac{z-z_{k}}{\Delta_{z}}\right) \beta\left(\frac{t-t_{l}}{\Delta_{t}}\right) \mathbf{p}_{i, j, k, l},
$$

where $\mathbf{x}=(x, y, z), \beta(\cdot)$ is a $1 \mathrm{D}$ cubic B-Spline kernel, $\left\{x_{i}, y_{j}, z_{k}, t_{l}\right\}$ define a regular grid of $4 \mathrm{D}$ control points, and $\Delta_{x}, \Delta_{y}, \Delta_{z}, \Delta_{t}$ are the spacings between control points in each dimension. We can compute the acceleration $\mathbf{a}(\mathbf{x}, t ; \mathbf{p})$ as

$\mathbf{a}(\mathbf{x}, t ; \mathbf{p})=\frac{\mathrm{d} \mathbf{v}(\mathbf{x}, t ; \mathbf{p})}{\mathrm{d} t}=\sum_{i, j, k, l} \beta\left(\frac{x-x_{i}}{\Delta_{x}}\right) \beta\left(\frac{y-y_{j}}{\Delta_{y}}\right) \beta\left(\frac{z-z_{k}}{\Delta_{z}}\right) \frac{\mathrm{d}}{\mathrm{d} t} \beta\left(\frac{t-t_{l}}{\Delta_{t}}\right) \mathbf{p}_{i, j, k, l}$,

Acceleration is computed on all nodes of the mesh obtained from segmentation of biventricular heart.

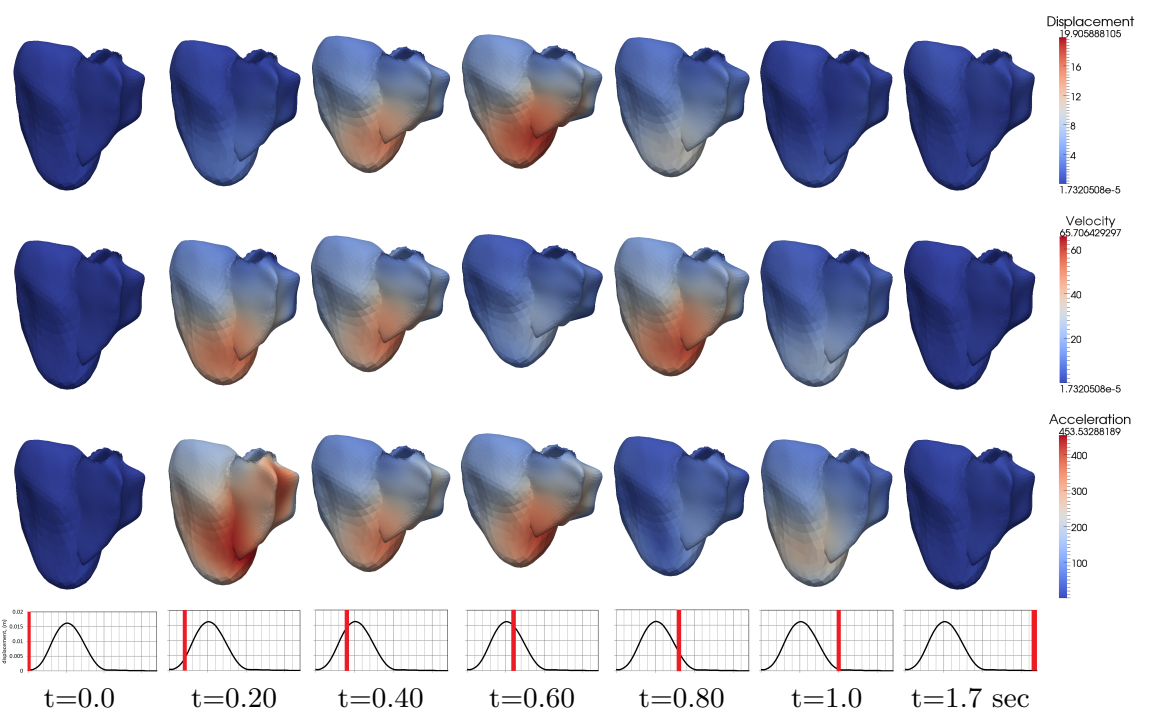

Fig. 3. Distribution of the displacement (upper row), velocity (middle row) and acceleration (lower row) magnitudes during the heart cycle. Units for displacement, velocity and acceleration are $m, m / s$ and $m / s^{2}$, respectively 


\section{Results}

\subsection{Displacement, velocity and acceleration in heart}

Fig.3 shows the magnitude of the estimated displacement, velocity and acceleration of the myocardium, along the cardiac cycle. These results are computed based on the image acquisition with frame rate $21.7 \mathrm{fps}$. Results were coherent with the echocardiographic images, and the program used for the phantom: stationary position in the cycle before $t=0.20 \mathrm{sec}$ and after $t=1 \mathrm{sec}$; higher values at the apex with respect to the basal level, and at end-diastole. Values change smoothly both in time and space due to the use of the TDFFD algorithm.

Results are detailed in Fig. 4 for four locations covering the myocardium from base to apex. The four points correspond to epicardium on apex, apical inferior, mid inferior and basal inferior section of the AHA 17-segment model, which are labeled with 17, 15, 10 and 4 respectively. Displacement and acceleration components in radial, circumferential and longitudinal directions are presented in Fig. 4(a) and (b), respectively. Realistic displacements are observed: sinus waveform as programmed along the radial direction at the apex (note that due to the phantom materials and setup, radial contraction is not necessarily guaranteed in the other regions); almost no circumferential motion; and decreasing longitudinal motion from apex to base.
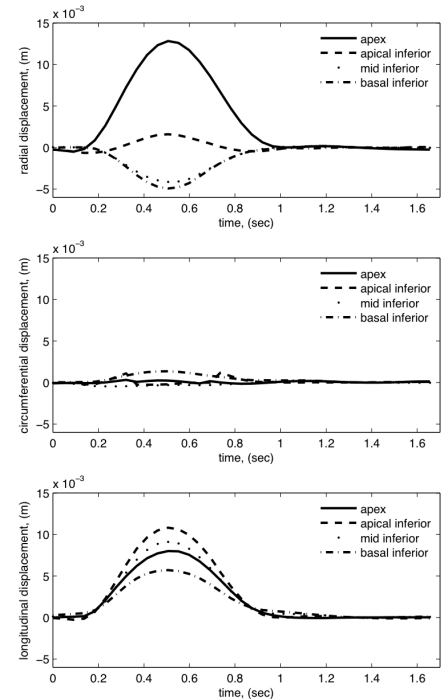

(a)
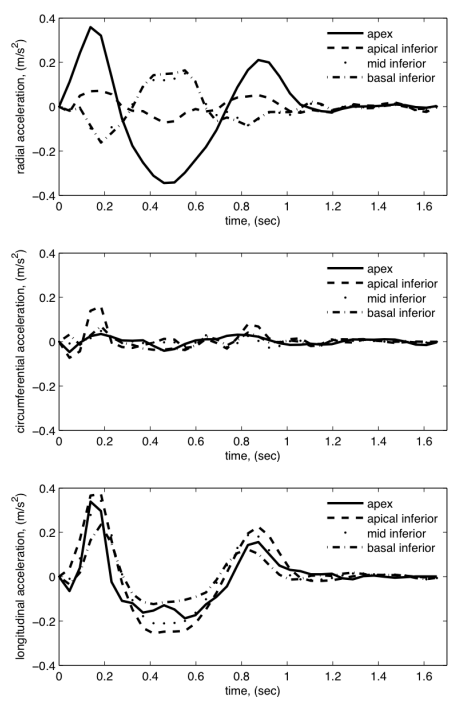

(b)

Fig. 4. (a) Displacement and (b) acceleration in radial, circumferential and longitudinal directions on four regions on epicardium during heart cycle. 


\subsection{Displacement and acceleration in apex}

Solid curve in Fig. 5(a) illustrates the recovered displacements at the apex from TDFFD algorithm. The presented curve is the average magnitude of displacement over the 17th AHA segment, along the cardiac cycle. Dashed line in this figure is the configured displacement function from equation (1). TDFFD follows the configured displacement function with mean squared error (MSE) of $5.15 \times 10^{-6} \mathrm{~m}^{2}$. The magnitude of peak displacement was $16.2 \mathrm{~mm}$, which corresponds to $19 \%$ relative error with respect to the settings of the phantom actuator.

The solid line in Fig. 5(b) shows the cardiac acceleration estimated by TDFFD at the apex. Dashed line in this figure shows the second analytic derivative of displacement function in equation (2). The MSE error from this estimation was found to be $0.011 \mathrm{~m}^{2} / \mathrm{s}^{4}$. PEA was measured $0.285 \mathrm{~m} / \mathrm{s}^{2}$, which shows $20.4 \%$ error with respect to the ground truth peak acceleration $0.358 \mathrm{~m} / \mathrm{s}^{2}$ computed from equation (2).

\subsection{Effect of frame rate}

We performed and experiment where the frame rate was decreased from 21.7 to $11.9 \mathrm{fps}$ to study the impact of temporal resolution on the image based estimations. Fig. 5(c) shows the radial acceleration at the same point in the apex computed from high and low frame rate imaging data. Acceleration magnitude has considerably changed under the effect of a lower temporal resolution. PEA from the low frame rate images was measured $0.235 \mathrm{~m} / \mathrm{s}^{2}$, which under-estimates this value by factor of $34.3 \%$ and $17 \%$ with respect to the ground truth data and estimation from high frame rate images, respectively.

\section{Discussion}

Computing myocardial acceleration from medical images has several advantages over other existing techniques: the measurement is non-invasive and provides information at any location of the myocardium, and not at a single point. Furthermore (not demonstrated here) accelerometer sensors are sensitive to environmental factors such as gravity [6], which is not the case of image-based data.

In this study, we presented a method for estimating cardiac acceleration directly from temporal sequences of images. We used a non-rigid registration algorithm (TDFFD) to track the myocardium along the cycle, which has several advantages: (i) it provides differentiable velocities, necessary to the computation of acceleration; (ii) output data is smooth in both time and space, which may prevent from artifacts due to low image quality and low temporal resolution.

Fig. 5 shows the performance of this method for tracking the sinus waveform displacement. The error on peak displacement was $19 \%$ and PEA error was $20.4 \%$ and $34.3 \%$ using imaging data with frame rate 21.7 and $11.9 \mathrm{fps}$, respectively. Some possible reasons for this error can be the sensitivity of the measurements to image quality and the relatively low temporal resolution. This hypothesis is 


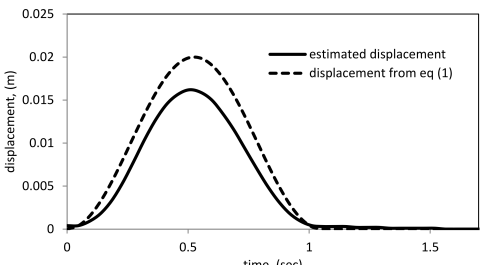

(a)

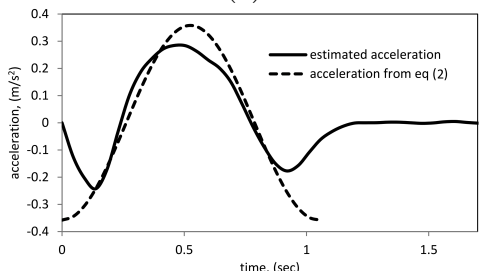

(b)

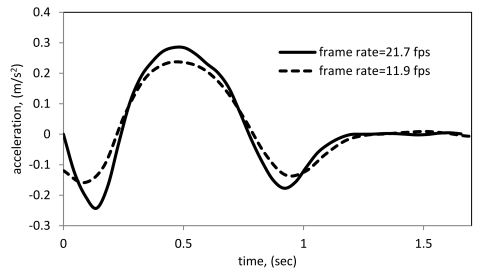

(c)

Fig. 5. (a) Estimated displacement (solid line) versus analytical plot of equation (1) (dashed lines), (b) estimated acceleration (solid line) compared to analytical plot of equation (2) (dashed lines), and (c) estimated acceleration from image data of frame rate $21.7 \mathrm{fps}$ (solid line) comapred to that of image data with frame rate $11.9 \mathrm{fps}$ (dashed line).

reinforced by our experiment on lower frame rate sequences, which results in a drop-off of the acceleration accuracy.

In our study, the acceleration was computed at every spatiotemporal location, but validation against ground truth was only possible at apex. The use of a FFDbased registration scheme, which intrinsically contains spline interpolation, may result in oversmoothing of the estimated curves. While this is not desirable for real applications measuring faster motion and deformation patterns, it also prevents the acceleration from being too noisy and therefore unexploitable. This is one limit of our study, that one may consider less relevant in the future with the availability of higher frame rates for 3D echocardiography.

Sonomicrometry cristals may be used to provide ground truth at other specific locations, but this was not retained in our study due to the risk of damage 
to the phantom material. Tagged-MRI is another option usually used as a surrogate of ground truth, but this would require a MR compatible phantom, which is not the case of the one we have.

\section{Conclusion}

We tested a method for estimating myocardial acceleration directly from image sequences, using non-rigid registration techniques (TDFFD), which has the advantage of providing spatiotemporally smooth velocities, and allows to compute acceleration directly from them. Experiments on a realistic phantom showed feasibility of recovery of acceleration profiles using this method. It suggests a non-invasive technique to measuring the cardiac acceleration that might be used to improve the monitoring of cardiac mechanics and optimization of CRT.

\section{Acknowledgements}

This work has been partially supported by the Spanish Ministry of Economy and Competitiveness (Ref. TIN2012-35874 and TIN2011-28067) and by the Spanish Ministry of Science and Innovation under the cvREMOD CENIT Project (CEN20091044). Authors acknowledge the support from Xavier Planes (UPF, Barcelona, Spain), Marta Sitges (HCPB, Barcelona, Spain) and Alejandro F. Frangi (U of Sheffield, UK).

\section{References}

[1] P. Bordachar, L. Labrousse, S. Ploux, J. B. Thambo, S. Lafitte, P. Reant, P. Jais, M. Haissaguerre, J. Clementy, and P. Dos Santos, "Validation of a new noninvasive device for the monitoring of peak endocardial acceleration in pigs: implications for optimization of pacing site and configuration," Journal of Cardiovascular Electrophysiology 19(7), pp. 725-729, 2008.

[2] P. S. Halvorsen, E. W. Remme, A. Espinoza, H. Skulstad, R. Lundblad, J. Bergsland, L. Hoff, K. Imenes, T. Edvardsen, O. J. Elle, and E. Fosse, "Automatic real-time detection of myocardial ischemia by epicardial accelerometer," $J$ Thorac Cardiovasc Surg 139(4), pp. 1026-1032, 2010.

[3] P. P. Delnoy, E. Marcelli, H. Oudeluttikhuis, D. Nicastia, F. Renesto, L. Cercenelli, and G. Plicchi, "Validation of a peak endocardial acceleration-based algorithm to optimize cardiac resynchronization: early clinical results," Europace 10(7), pp. 801-808, 2008.

[4] N. Olsen, R. Mogelvang, C. Jons, T. Fritz-Hansen, and P. Sogaard, "Predicting response to cardiac resynchronization therapy with cross-correlation analysis of myocardial systolic acceleration: a new approach to echocardiographic dyssynchrony evaluation," Journal of the American Society of Echocardiography 22(6), pp. 657-664, 2009.

[5] E. W. Remme, L. Hoff, P. S. Halvorsen, E. Naerum, H. Skulstad, L. A. Fleischer, O. J. Elle, and E. Fosse, "Validation of cardiac accelerometer sensor measurements," Physiological Measurement 30(12), pp. 1429-1444, 2009. 
[6] E. Remme, L. Hoff, P. Halvorsen, A. Opdahl, E. Fosse, and O. Elle, "Simulation model of cardiac three dimensional accelerometer measurements," Medical Engineering and Physics 34(7), pp. 990-998, 2012.

[7] A. Pashaei, G. Piella, X. Planes, N. Duchateau, T. M. de Caralt, M. Sitges, and A. F. Frangi, "Image based cardiac acceleration map using statistical shape and $3 \mathrm{~d}+\mathrm{t}$ myocardial tracking models; in-vitro study on heart phantom," in SPIE Medical Imaging, SPIE, (Florida USA), 9-14 Feb 2013.

[8] M. De Craene, G. Piella, O. Camara, N. Duchateau, E. Silva, A. Doltra, J. D'hooge, J. Brugada, M. Sitges, and A. F. Frangi, "Temporal diffeomorphic freeform deformation: application to motion and strain estimation from $3 \mathrm{~d}$ echocardiography," Medical Image Analysis 16(2), pp. 427-450, 2012.

[9] H. C. van Assen, M. G. Danilouchkine, A. F. Frangi, S. Ordas, J. J. M. Westenberg, J. H. C. Reiber, and B. P. F. Lelieveldt, "SPASM: a 3D-ASM for segmentation of sparse and arbitrarily oriented cardiac MRI data," Medical Image Analysis 10(2), pp. 286-303, 2006.

[10] R. Byrd, P. Lu, J. Nocedal, and C. Zhu, "A limited memory algorithm for bound constrained optimization," SIAM Journal on Scientific Computing 16(5), pp. 1190-1208, 1995.

[11] M. Cerqueira, N. Weissman, V. Dilsizian, A. Jacobs, S. Kaul, W. Laskey, D. Pennell, J. Rumberger, T. Ryan, M. Verani, et al., "Standardized myocardial segmentation and nomenclature for tomographic imaging of the heart a statement for healthcare professionals from the cardiac imaging committee of the council on clinical cardiology of the american heart association," Circulation 105(4), pp. 539-542, 2002. 